\title{
XIII.
}

\section{Zur Frage der Gallenfarbstoffbildung aus Blut.}

\author{
II. Mittheilung ${ }^{1}$ ).
}

Von

\section{Prof. Dr. Theodor Brugsch,}

nach Versuchen von Dr. Yoshimoto.

Es ist "eine alte Forderung der Pathologie, dass zwischen dem Blutfarbstoff und dem Gallenfarbstoff ein naher genetischer Zusammenhang bestehen müsse" ${ }^{2}$ ).

Dieser genetische Zusammenhang zwischen Gallenfarbstoff und Hämatin würde sich vielleicht nach folgender Gleichung vollziehen:

$$
\mathrm{C}_{32} \mathrm{H}_{32} \mathrm{~N}_{4} \mathrm{O}_{4} \mathrm{Fe}+2 \mathrm{H}_{2} \mathrm{O}-\mathrm{Fe}=\mathrm{C}_{32} \mathrm{H}_{36} \mathrm{~N}_{4} \mathrm{O}_{6} \text {. }
$$

Die folgenden Versuche sind unternommen, um diese Frage zu lösen. Es musste dabei das Problem gelöst werden, quantitativ die Gallenfarbstoffausscheidung beim Thiere mit künstlicher Gallenfistel und unterbundenem Ductus choledochus zu verfolgen.

\section{Methodik des Versuches.}

Es wurde zuerst eine $12,800 \mathrm{~kg}$ wiegende, leicht katheterisirbare Hündin ausgesucht. Am 4. Mai 1909 wurde der Hündin eine Gallenfistel angelegt. Vom dritten Tage nach der Operation an wurden Harn, Koth und Galle sorgfältig angesammelt. Zu diesem Zwecke wurde die Hündin in einen sorgfältig gereinigten Käfig gebracht und der Mündung der Gallenfistel eine grössere Menge sterilisirter Gaze vorgelegt und darüber ein mit Gummi gefütterter Verband angebracht, um die secernirte Galle verlustlos zu sammeln. Um den Verband ordentlich an Ort und Stelle zu halten, wurde die Hündin während des ganzen Versuches Tag und Nacht an einem breiten weichen Tuche aufgehängt. Der Harn wurde stets durch Katheterismus entleert. Der Verbandwechsel und die Kothaufsammlung geschah jeden Tag zu einer bestimmten Zeit.

Der Versuch dauerte 29 Tage; wir theilen die ganze Versuchszeit in vier Perioden, und zwar die

1) I. Mittheilung s. Tsuchiya, Lur Frage der Urobilinausscheidung. Diese Zeitschrift. Bd. V.

2) Nencki u. Zaleski, Arch. f. exper. Path. u. Pharm. Bd. 18. S.401. (1884.) 


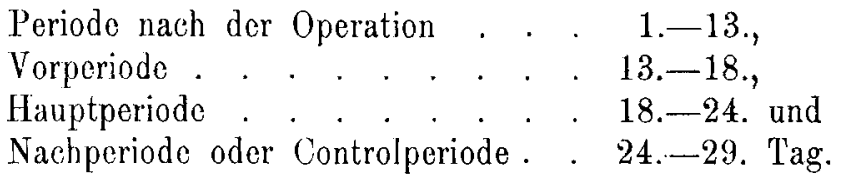

In der Hauptperiode wurde dem Versuchsthier subeutan jeden Tag 12,5 cem einer 0,4 proc. alkalischen Lösung von salzsaurem Hämin $(=0,05 \mathrm{~g}$ Hämin) eingespritzt.

Das im Versuche angewandte salzsaure Hämin wurde nach der Mörner'schen Vorschrift ${ }^{1}$ ) aus Hammelblut dargestellt. Die Häminlösung wurde jeden Tag in folgender Weise friseh bereitet: $0,1 \mathrm{~g}$ Hämin wurde in genau $25 \mathrm{ccm}$ Wasser, welches vorher mit 2-3 Tropfen Normalnatronlauge alkalisch gemacht war, aufgelöst.

\section{Entersichung des Harns.}

Die saure, ätherisch-alkalische Urobilinlösung wurde nach dem Vorschlage von Friedrich Müller und Huppert ${ }^{2}$ ) in folgender Weise dargestellt: $100 \mathrm{cem}$ Harn $(50 \mathrm{ccm}$, falls der Harn zu concentrirt war) wurde mit ca. $30 \mathrm{ccm}$ einer Misehung ( $1 \mathrm{Vol}$. gesättigter Chlorbariumlösung und 2 Vol. gesättigten Barytwassers) behandelt, wodurch dic Harnsäure und das Hämatoporphyrin vollständig entfernt wird. Man filtrirt den Niederschlag und das Filtrat wird mit concentrirter Natriumsulfatlösung versetzt, wobei das überschüssige Barium als Bariumsullat gefüllt wird. Man filtrirt wicderholt, bis das Filtrat ganz klar wird. Wenn das Filtrat zu stark alkalisch ist, so setzt man verdünnte Schwefelsäure tropfenweise $z u$, bis die Flüssigkeit fast neutral wird. Nun setzt man zu der Flüssigkeit soviel Ammoniumsulfat hinzu, bis cin ganz feiner, gelb-bräunlichflockiger Niederschlag entsteht. Man lässt das Gemisch bis zum nächsten Morgen stehen und filtrirt es mit einem troekenen Filter ab, wäscht es einige Male mit gesättigter Ammoniumsulfatlösung, trocknet den Filter sammt Niederschlag an der Luft vollständig. Dieser urobilinhaltige Niederschlag sammt Filter wird in cinem Rundkolben mit aufgesetztem Rückflusskühler mit einem Gemisch von Aether und absolutem Alkohol ( 1 Vol. : 2 Vol.) 6-8 Stunden lang extrahiert, wobei das Urobilin in die Flüssigkeit übergeht. Nach der Abkühlung wird das Extract durch einen kleinen trockenen Filter abfiltrirt, das Filtrat auf dem Wasserbade auf weniger als $25 \mathrm{ccm}$ eingeengt. Die concentrirte Urobilinlösung wurde in einem Messkolben auf $25 \mathrm{ccm}$ aufgefüllt und dann spectrophotometriseh untersucht.

Zu diesem Zwecke wurde der von König-Martens'sche Spectrophotometer in Anwendung genommen. Ueber die Construction und die Gebrauchsweise des Spectrophotometers vergleiche dic Arbeiten von F. F. Martens ${ }^{8}$ ) und 1. T'suchiya. ${ }^{4}$ Die Berechnung geschah wie bei Tsuchiya.

1) Handbuch der chemischen Analyse von IIoppo-Seyler. 1909. S. 358.

2) Ebendaselbst. 1909. S. 379 .

3) Verhandlungen der deutschen physiologischen Gesellschait. I. Jahrg. No. 15.

4) 1. c. 


\section{Untersuchung des Kothes.}

Die quantitative Bestimmung des Urobilins im Kothe wurde folgendermaassen ausgetührt. Die in 24 Stunden entleerte Kothmenge wurde mit dem ungefähr $2-3$ fachen Volumen von 93 proe. Alkohol gut durchgerührt und bei Zimmertemperatur 10-12 Stunden lang stehen gelassen. Nach dem Ablauf der Zeit wird der alkoholische Extract abfiltrirt, man wäscht den Filterinhalt einige Male mit Alkohol aus. Der alkoholische Urobilinextract wird nun auf dem Wasserbade auf ca. $10 \mathrm{ccm}$ eingedampit. Man giesst dann in die Schale ca. 50-100 ccm Wasser hinzu und filtriert die Flüssigkeit auf einem feuchten Filter ab. Das Filtrat wird nun mit der 3-4 fachen Menge Petroläther in einem Scheidetrichter stark ausgesehüttelt, wobei andere Farbstoffe, Fett, Indol und Skatol etc. entfernt werden. Die Darstellung der sauren, ätherisch-alkoholischen Urobilinlösung aus dieser Flüssigkeit geschah genau so wie beim Harne; die quantitative Untersuchung durch Spectrophotometer.

\section{Untersuchung der Galle.}

Die mit der Galle durchtränkte Gaze wurde der Luft und dem Licht einige Tage gut ausgesetzt, wobei das intensiv gelbe Bilirubin zum grünen Biliverdin oxydirt wird. Diese grünlich gefärbte Gaze wird in einen 11/2 Liter haltenden Rundkolben mit Rückflusskühler gebracht und mit $500-600 \mathrm{ccm}$ Alkohol (93 proc.) und einigen Cubikcentimetern verdünnter Schwefelsäure über 10 Stunden extrahirt.

Danach wurde die Gaze abgepresst und einige Male mit Alkohol gewaschen und wieder abgepresst, bis die Gaze farblos wurde.

Aus diesem sauren alkoholischen Gallenfarbstoffextracte wurde zunächst der Farbstoff nach folgendem Verfahren rein dargestellt:

Man filtrirt das Extract, dampft es auf dem Wasserbade ein, bis der Rückstand als eine dunkelgrüne syrupöse Masse binterlassen wird. Nach dem völligen Erkalten wurde der syrupartige Rückstand mit concentrirter Schwefelsäure (1,84 spec. Gew.) wieder aufgelöst. Man setzt nun ganz vorsichtig mittels einer Spritzflasche unter tüchtigem Umrühren tropfenweise Wasser $\mathrm{za}$, bis zuerst ein ganz feiner Niederschlag entsteht und dann an der Berührungslinie der Schale mit der Farbstofflösung eine dunkelgrüne, klebrige Masse zum Vorschein kommt. Man lässt nun das Gemisch erkalten und bis zum nächsten Morgen stehen. Am folgenden Morgen giesst man wieder Wasser in grösserer Menge hinzu, wobei schöne grossflockige grüne Niederschläge entstehen. Man filtrirt den Niedersehlag $a b$, wäscht ihn mit Wasser so lange $a b$, bis im Waschwasser keine Schwefelsäurereaction mehr vorhanden ist (genügende Waschung ist wesentlich), trocknet ihn an der Luft gut, extrahirt den Niederschlag sammt Filter mit absolutem Alkohol (99,8 proc.) in einem Rundkolben, filtrirt das Extract, verdunstet es auf dem Wasserbade, trocknet es an der Luft, und so bekommt man schliesslich eine glänzende, dunkelgrüne, amorpbe Substanz. Diese Substanz löst sich in Alkohol ${ }^{1}$ ) sehr leicht auf, dagegen nicht in Wasser, Aether und Chloroform.

1) Diese alkoholische Lösung ist frisch grasgrün, durchsichtig, beim langen A ussetzen an Luft und Licht wird sie allmählich gelbbräunlich. 
Bei der Einwirkung eines reducirenden Körpers, z. B. Schwefelammon, läsșt sie sich leicht zu Bilirubin bezw. Urobilin reduciren, sie löst sich in concentrirter Scliwefelsäure auf und beim Wasserzusatz scheidet sie wieder unverändert ab. Ihre Lösungen zeigen keine Absorptionsstreifen.

Mit diesem Gallenfarbstoff wurde nun eine 0,05 proc. saure alkoholische Lösung bereitet und diese Lösung als die normale Lösung für die quantilative Gallenfarbstoffbestimmung benutzt.

Bei allen alkoholischen Farbstoffextracten ist allerdings der Farbton nicht immer ganz gleich, und es ist erforderlich, manchmal den Farbton der Normallösung zu ändern, wodurch erst die exacte colorimetrische Farbstoffbestimmung ermöglicht wird. $\mathrm{Zu}$ diesem $\mathrm{Zwecke}$ haben wir zwei gleichprocentige $(0,05$ proc.) aber von einander ganz verschiedene farbtonhaltige Normallösungen bereitet (eine, welcke grasgrün und eine, welche bräunlicher ist). Bei der Combination dieser beiden Lösungen erhält man dann eine Normallösung mit jedem farbentonhaltigen alkoholischen Extract.

Als Colorimeter benutzten wir die Plesch'schen colorimetrischen Apparate (am besten eignet sich dazu der Plesch'sche Kolbenkeilcolorimeter), deren Schraubenzahl direct den Procentsatz der betreffenden Lösung gegen die normale zeigt.

War die Concentration des alkoholisehen Extracts im Vergleich zu der Normallösung zu stark, so wurde sie je nach der Concentration auf das 2,3 bis 5 fache verdünnt.

Die Berechnung der Tagesfarbstoffmenge ist einfach, z. B. bei einem

Tabelle I.

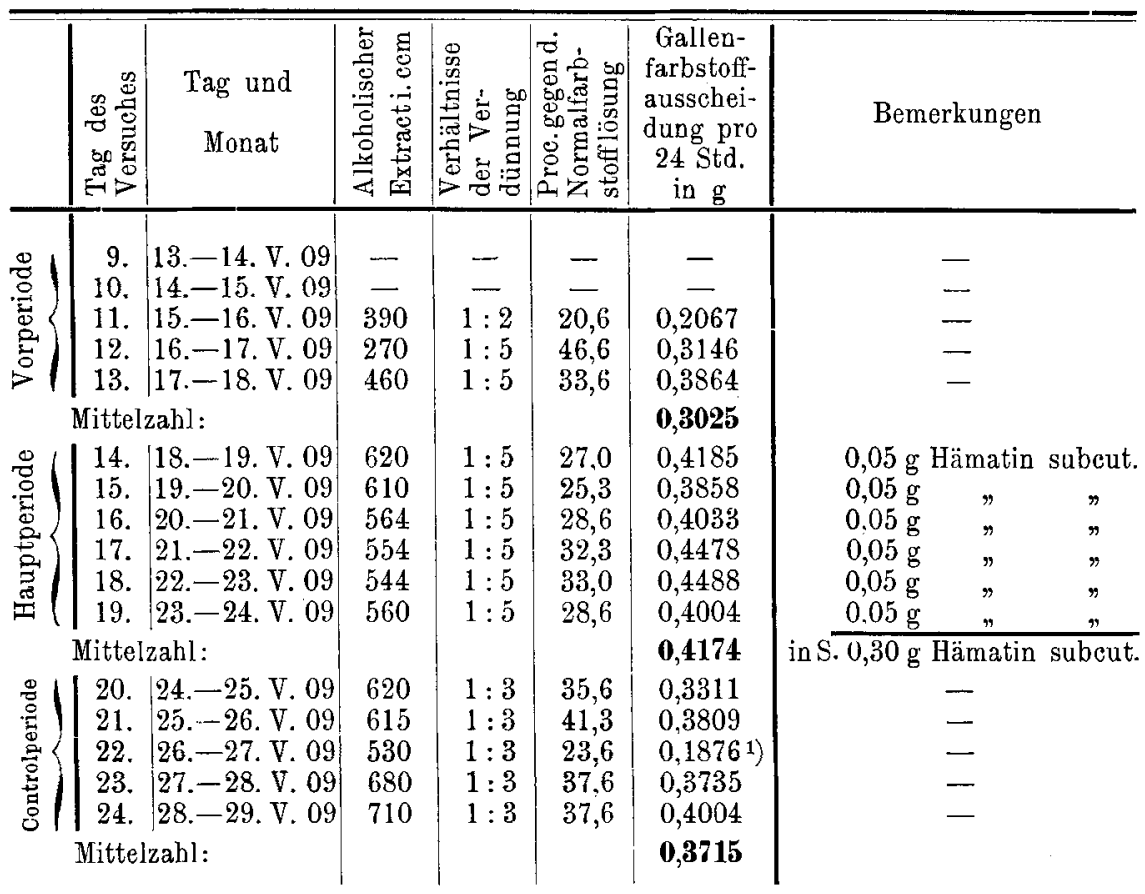

1) Durch Verlust fehlerhafter Werth! 
Versuchstage (dem 18.-19. V.) betrug der alkoholische Extract $620 \mathrm{ccm}$, die Verdünnung war 5 fach und der Procentsatz 27.

Wir erhalten dann:

$$
0,05 \times \frac{27}{100} \times 620 \times 5=0,4185 \mathrm{~g} .
$$

Was nun die Resultate der vorliegenden Arbeit anbetrifft, so sind diese in den Tabellen I und II angeordnet.

Tabelle II.

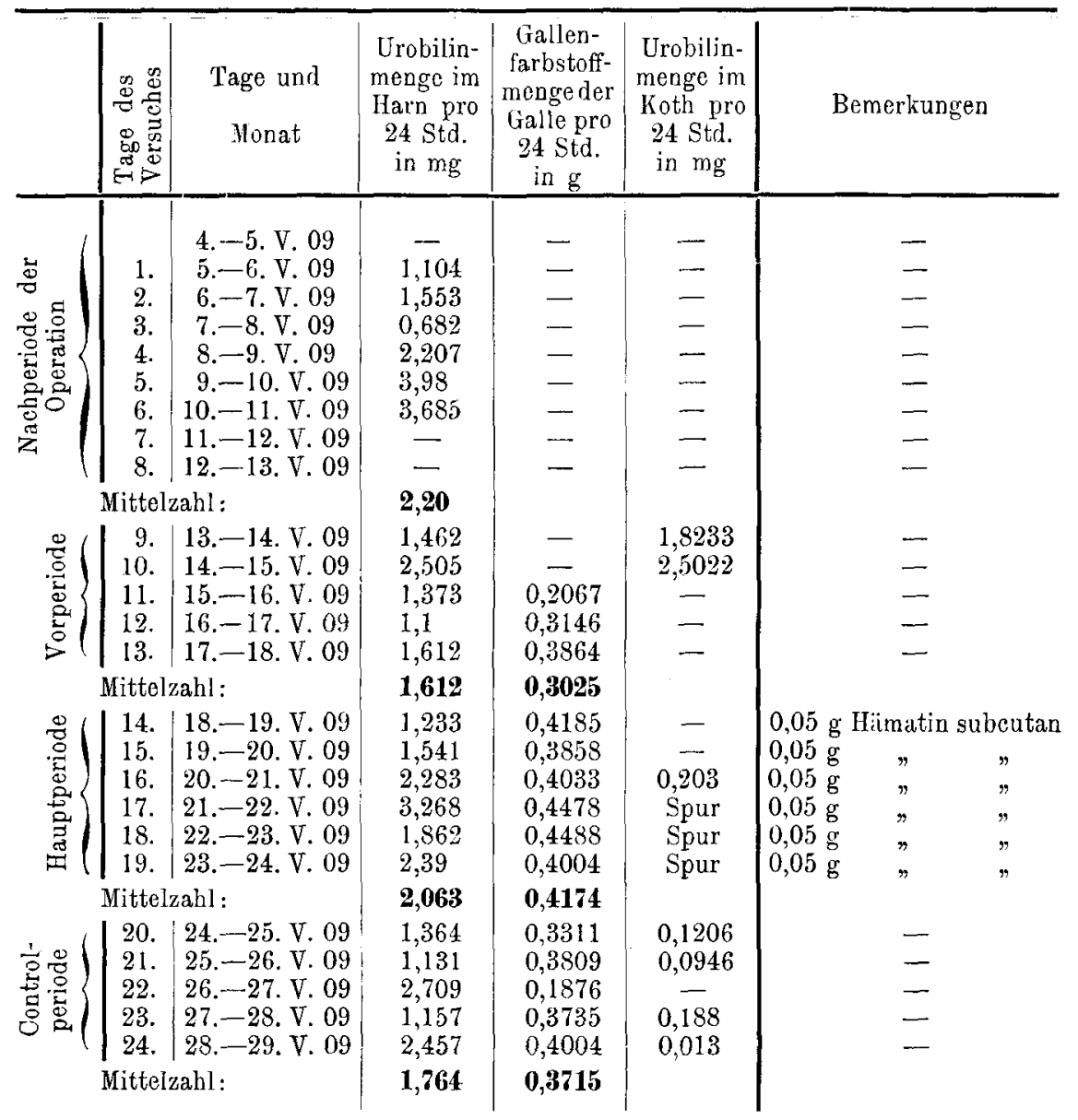

Die Resultate sind eindeutig.

Was zunächst die Gallenfarbstoffmende anbelangt, so erscheinen die täglichen Werthe in der Nachperiode im Ganzen constant, in der Vorperiode weniger constant, indessen doch so, dass man einen annähernden Mittelwerth von etwa $0,37 \mathrm{~g}$ Gallenfarbstoffausscheidung berechnet auf Biliverdin annehmen kann. In der Hauptperiode erhöht sich dieser Gallenfarbstoffwerth, und zwar durchschnittlich täglich um $0,04 \mathrm{~g}$. Da nun täglich $0,05 \mathrm{~g}$ Hämin subcutan eingespritzt wurden, müssen wir annehmen, dass fast quantitativ das Hämatin in Gallenfarbstoff übergegangen ist. Als Urobilin dürften nur Spuren in den Harn übergegangen sein. Der 
644 Brugsch-Yoshimoto, Zur Frage der Gallenfarbstolfbildung aus Blut.

mittlere Werth der Hauptperiode beträgt $2,0 \mathrm{mg}$, gegenüber $1,6 \mathrm{mg}$ und $1,7 \mathrm{mg}$ der Vor- und Nachperiode.

Es ist somit der stricte Beweis geliefert, dass Hämatin die Quelle der Gallenfarbstoffbildung ist, und zwar scheint schon nach diesen Vorsuchen die Gleichung zuzutreffen:

$$
\mathrm{C}_{32} \mathrm{H}_{32} \mathrm{~N}_{4} \mathrm{O}_{4} \mathrm{Fe}+2 \mathrm{H}_{2} \mathrm{O}-\mathrm{Fe}=\mathrm{C}_{32} \mathrm{H}_{36} \mathrm{~N}_{4} \mathrm{O}_{6} \text {. }
$$

Wir versagen es uns, an dieser Stelle schon die Grösse des täglichen Blutzerfalls auf Grund der oben gewonnenen Erkenntniss und der Kenntniss der täglichen Gallenfarbstoffausscheidung zu berechnen. 